\title{
The Intensity of Electromagnetic Wave Detection Robot based on ARM
}

\author{
Bin Huang, Wenbo Zhu,Licheng Liu \\ Logistics Engineering College, Wuhan University of Technology, Wuhan, 430063, China
}

\begin{abstract}
Keywords:electromagnet radiation; monitoring; robot.
\end{abstract}
\begin{abstract}
Aiming at the increase of electronic products brought about by the hazards of electromagnetic radiation intensity of an electromagnetic wave, designed a time for detection of people within the scope of activities to re-mind people to exceed the standard, in order to prevent the electromagnetic wave radiation inspection robot for hazards. It can be based on the strong electromagnetic wave signal detection, to find its location, which can help people make reference in the choice of housing, the government planning of residential areas. At the same time, it also can be the key unit of strict control of electromagnetic radiation. The robot is widely popularized in the occasions with electromagnetic radiation it has good portability and wide market application prospect.
\end{abstract}

\section{Introduction}

With the development of science and technology, TV, refrigerator, washing machine, microwave oven, electronic products such as mobile phone ,computer, various entered the people's work, study, life and production, and brings convenience to the people. But the electromagnetic release hazards to human is also gradually increasing.

According to the electromagnetic radiation hazards, the project team to design a can always detect electromagnetic wave people activities within the scope of the radiation intensity to remind people, thus preventing electromagnetic wave radiation damage is a kind of detection device of robot, it can through the detection of strong electromagnetic wave signal, find strong electromagnetic wave emission source location. To help people in the choice of housing, and the government in the planning of residential area to make reference, let people try to avoid strong electromagnetic wave place [4]. Key units at the same time the robot on electromagnetic radiation of strict control, once exceeded, regulators may be given heavy penalties for excessive radiation unit, and ordered its deadline rectification [5]. The machine in the electromagnetic radiation with occasions can also be carried out a large number of promotion, has good portability and wide market application prospect.

\section{Overall design}

This monitoring robot is mainly composed of a detection module, signal processing module, a supporting mechanism, a lifting mechanism, mobile mechanism and other parts, in order to realize the automatic wide scope of application, the basic concept of detection. The detection module, signal processing module is the core part of the robot, it can always detect electromagnetic wave intensity and determine the direction. At the same time, the design of lifting mechanism to solve the problem of robot driving on different road surface. It can realize the following functions:

(1)Electromagnetic wave rapidly accurately detect emission source emission is also a specific range of band of electromagnetic wave, electromagnetic wave signal intensity distribution including the location of the robot.

(2) Can be calculated by the ARM microcontroller program to the strongest signal position in the range direction (if any such as signal towers near the launch tower and the robot can measure distance), then goes the strongest electromagnetic wave direction, to find the strength of the strong electromagnetic wave region.

(3)The robot has to change the function of the foot with the wheels and tracks, two kinds of travel ways, which can adapt to different road surfaces. 


\subsection{Single chip microcomputer design detection control system based on the arm}

The first is its functional analysis:

The detection control part mainly realizes the control detects a current position of a particular band signal strength and output, and then control the car travel to find the strongest signal in near space position, issued at the same time, voice alarm and other functions.

The system is opened, the input signal by party band wanted to test, through the sensor receives the electromagnetic wave intensity of a specific band of the current location, and then through the A/D conversion chip for signal acquisition, and then quantizing and coding can be turned into a digital quantity. System identification is within the system on the basis of TDOA algorithm operation. Finally found the strongest and alarm [2]. Can switch mode, enter a value, when the current signal strength exceeds this value when the alarm. The process is shown in figure:

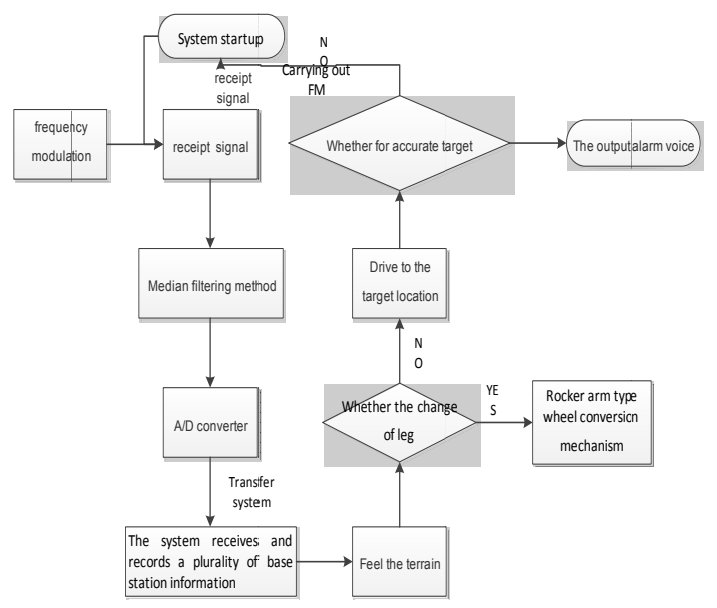

\subsection{Part of the hardware}

Figure 1 flow chart of system

This part is composed of a main controller, signal detection sensor, A/D converter module, and voice alarm part device four blocks.

the main controller

Use ARMJZ2440 development board, on the Internet can be easily downloaded about it related program template, and then through a simple modification according to their own needs to be possible; and it has multiple interfaces, IIC interface is rich in resources, can be very convenient to connect with the external module variety; we also can be easily carried out operation for it, we want to achieve the purpose of high reliability.

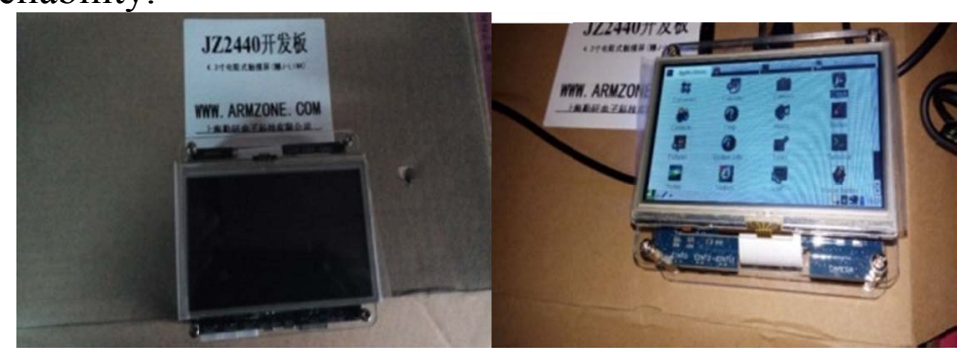

Figure 2 JZ2440 development boardFigure 3 Renderings with the system

The $\mathrm{A} / \mathrm{D}$ conversion module

The electromagnetic field is a physical field, consisting of the sum of the interdependence of electromagnetic and magnetic field. Both electromagnetic and magnetic field and electromagnetic field is formed reciprocal causation, Which produces an electric field when the magnetic field changes over time, the electric field and magnetic field changes over time .The electromagnetic wave is in high frequency electromagnetic oscillations, part of the energy from the spatial spread out general wave and magnetic wave formed by radiation, electromagnetic wave is a form of motion in electromagnetic fields. So on the detection of electromagnetic waves can be converted into the detection of electromagnetic field.

To this end, we designed a wireless signal receiving module, the diagram as follows: 
This module through the first antenna signal, con4 is connected with the single chip microcomputer can control the signal frequency band receiving, con5 bus is connected with the single chip microcomputer for control, signal received by an antenna through a relay, transformer rectifier tube in tube feet con2 output, then complete the reception of signals.

This module is applicable to amplitude modulation (AM) radio, FM (FM) radio, radio television and mobile phone base stations in high frequency $(10 \mathrm{kHz} \sim 2165 \mathrm{MHz})$ electromagnetic wave emission source etc.
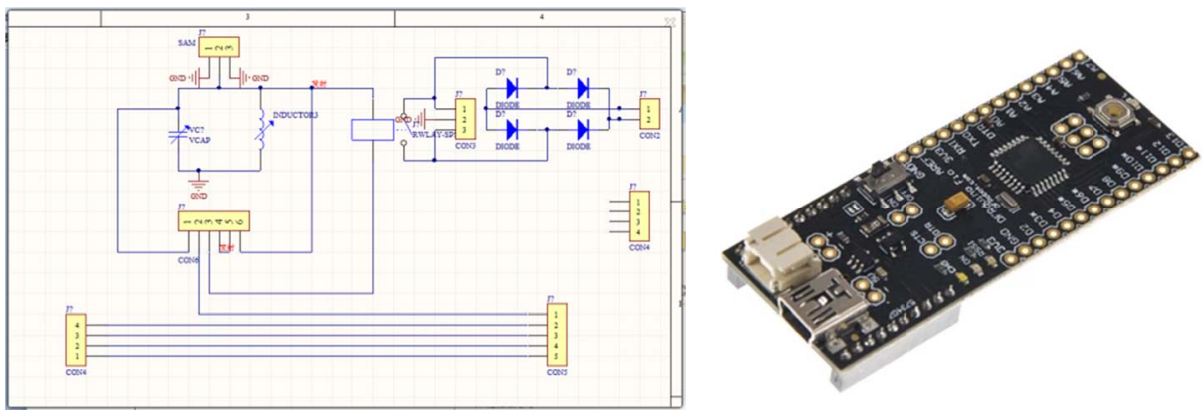

Figure 3 PCB receive module diagram Figure 4 DFRduino Player speech module

The speech control. Select DFRduino Player speech module as the alarm device, as shown in Figure 6, for a variety of prompt the process of exercise, music etc. Compared with other ordinary voice module, the module supports two kinds of interface, I2C and TTL level of serial port, convenient and Arduino, MCU and PC connection. Need to voice files placed in the SD card, play the corresponding speech files by sending the file name can be.

\section{3 part of the software}

The software part is mainly aimed at the operating system: want to let the rolbot can run, we need to use a JZ2440 development board to carry an operating system, burned into the development board to write the operating system, this project aims to use embedded Linux operating system. Because the embedded operating system in the optimization of system design, improve design efficiency, reduce the system design of repeated labor and improve the Portability has played a very positive role. The development of embedded Linux system is a huge project, after the hardware set up, mainly the following several basic steps:

The system boot program written in BootLoader

BootLoader (boot loader) is the first link of portable embedded system. BootLoader is the kernel of the operating system to start a small program that runs before, its main task is the beginning of the target board hardware, embedded system hardware resources to provide information on the board, and further loading and booting the embedded system firmware,. Therefore, the first step in embedded system transplantation is to transplant a suitable for hardware system of BootLoader.

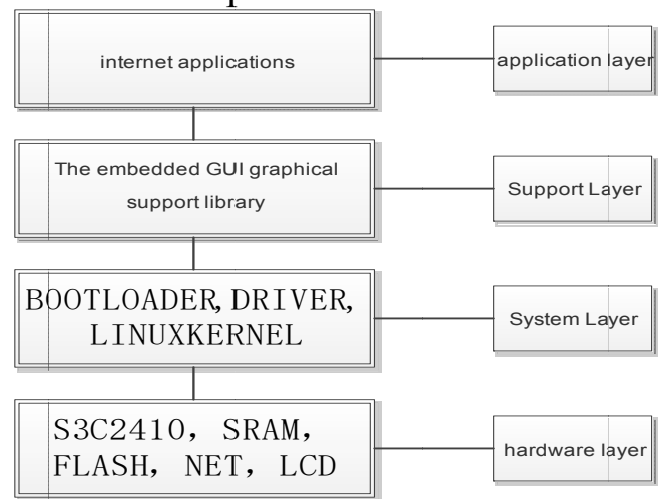

Fig.5 embedded Linux operating system development hierarchy

The Linux micro kernel compilation

The process of initialization

In addition, if you want to become a complete operating system and continue to maintain small must add hardware driver program, the hardware interface and application programs etc. Visible, the development of embedded Linux operating system has a certain level should according to the structure from the bottom up layer by layer, as shown in figure[1]. 


\section{Change of leg mechanism design part}

Design on the foot part, general design process we are as follows:

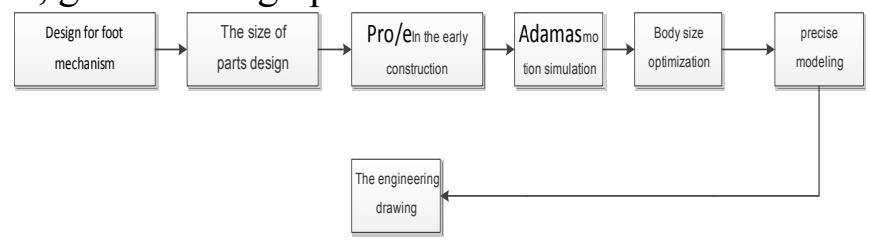

Fig.6 mechanism design flow chart

For the conversion of the track and wheels to realize the function, we design the content is, the four wheels of the robot is fixed to the chassis, let the track can lift, When the track surface below the wheel, the wheels to track conversion process, reverse, caterpillar to the wheels of the conversion process can be realized.

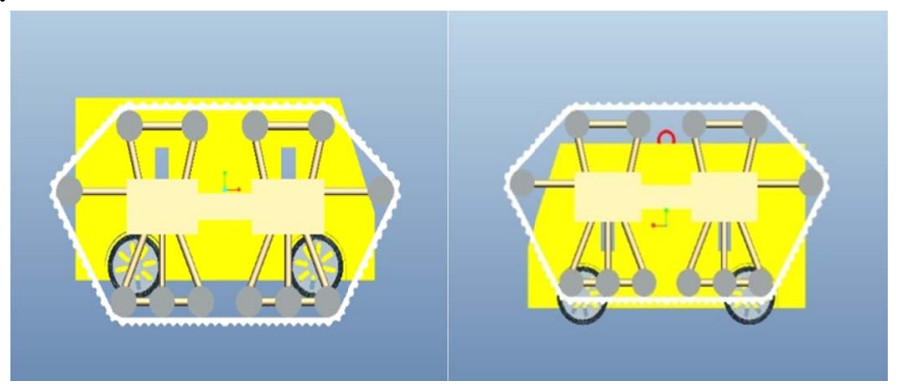

\subsection{Track support frame design}

Figure 7 track work

Figure 8 Track idle

In order to realize the lifting track, track fixed traditional way is not feasible, so we design a new type of supporting frame, the supporting frame is a symmetrical structure, contains two six corners supporting wheel system. When installed, will be a pair of track supporting frame arranged on the two sides of the car body. The reason that contains two supporting wheel system in a supporting frame is to maintain the balance of the moment.

Support each frame in the six corners of the supporting wheel system contains six track wheel meshing track wheel and track, similar to the mesh belt transmission.

Considering the problem of space frame supporting the overall, and the belt wheel speed synchronization problem, can't make each crawler wheel for driving wheel, so we set the supporting frame on the left and right most the crawler wheel for driving wheel, it can also maintain the balance of good.

In order to make the crawler can only move in a vertical direction, so we support center part sets up a slider, which is composed of H-shaped rod is connected with the supporting frame body, and the sliding block corresponding to the robot car at both ends of each set a slide.

The power source robot in general for rotary motion actuator or motor, in order to convert the rotary actuator for linear motion of the support frame, with the carriage slipway and the supporting frame of a slider, we applied a slider crank mechanism, the mechanism of the working state and the stable and has simple structure. We will crank and connecting rod set in the inside of the carriage, it is as if placed in the external, will be to track motion cause serious interference.

Because the slide Travel Co, so the movement of the slide blocks the existence of the limit position, when the slider is in the upper part, tracks in the idle state, when the slider is in the bottom, the crawler is in working state. That is to say, the rotary motion servo control crank must move within a certain angle, angle variation is constant. Therefore, in order to facilitate the servo control of the rotation angle of the crank and connecting rod length, calculated, crank, connecting rod set for the effects of crank and connecting rod, when in a straight line, the slider in the lower limit position of stroke, track work; when the crank and connecting rod slide block coincide, in the limit of travel position, track idle.

In other words, the rotation of the steering gear change Angle of 180 degrees, this is a special value is relatively easy to control, steering gear spindle rotation in one direction can achieve the 
reciprocating movement of the slider, but don't have to transform. At the same time, the structure design can guarantee mechanism motion no dead point, to prevent the steering gear being dead.

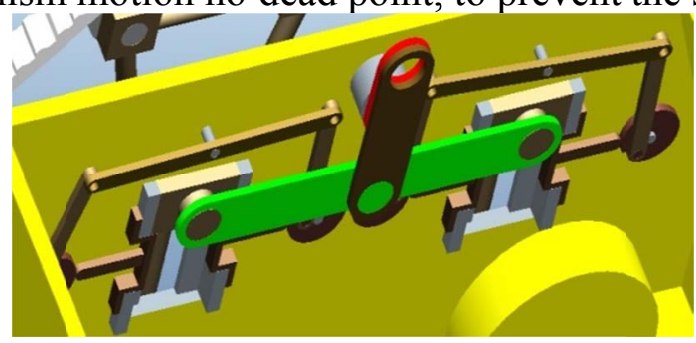

Fig. 9 limit of travel position slider

\subsection{Locking mechanism design}

Tracked generally work in a bumpy road, will shake up and down, simply by the crank connecting rod mechanism supporting force is not enough, and, when the crawler is idle, because of its integral part of greater weight on the crank, the connecting rod mechanism cannot to bear the all stress . In order to solve the above problems, we design a locking mechanism, no matter in what state is tracked, by locking mechanism responsible for supporting force. This greatly ensures the reliability and stability of crawler part.

The left and the right ends of slide way are respectively provided with a cam block, when the crawler is in idle state, the cam slider in the extended state, will support the slider support, bear crawler part of gravity; when the transition track to a working state, cam slide retracted, make its and slide plane coplanar, steering gear slider crank rotation followed by will be tracked down, slide, reaches the lower limit position of stroke, the steering gear to stop rotating; cam slide out again, so that you can speak support slide block is fixed at the lowest end, track work. When the conversion track by working state to the idle state, contrary to the above process.

In order to make the control cam slider extends and retracts (reciprocating linear motion), we designed a kind of cam mechanism. Characteristics of cam mechanisms are: that rotary motion can be converted into the reciprocating linear motion, and the cam can withstand the big radial force. In order to ensure the resetting cam slider, we will block a set of spring on the upper and lower ends, so it can guarantee the linkage lever on the slider can be close to the cam. Center of the cam and linkage rod axis collinear.

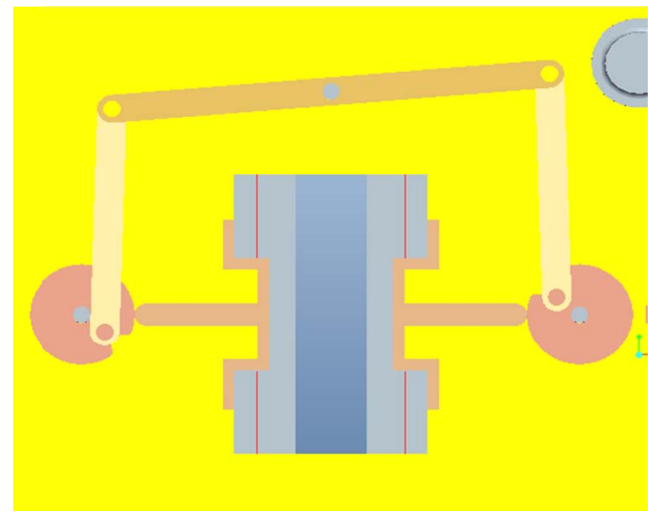

Figure 10 slide out

The circumference of cam is composed of three circular arcs. When the circular arc on cam slider of the linkage rod tangent with large arc, cam slide is pushed up out; when the tangent point by the large circular arc transition by small to medium-sized arc, because of the elastic force of the spring, the linkage rod close to the cam curve, slider slowly retracted; when the point of tangency to medium arc nadir, slide completely retract, and slide plane coplanar. So as to realize the conversion of extended and retracted cam slide.

Due to a slide system corresponds to about two of a cam, in order to save costs, the ideal situation is, a driving force input can make two cam rotate at the same time. In order to achieve this purpose, we designed a four cam linkage mechanism, its working principle is similar to the four connecting rods, and initial maintain symmetry component. 
The center part of rod one is connected with the steering spindle, as a power input. Rod one is hinged with the rod two and rod three in the left and right ends, rod two and rod three respectively hinged with the other ends around a point on the cam[3].

When the cam slider is in the retracted state, left and right cam center and its respective hinge joints and the linkage rod collinear, at this time, the four cam linkage mechanism is in the shape of a rectangle; the steering rod rotate a small angle, drive the driven rod one rotate, then the rod two and rod three translational motion, so as to drive the left and right of the cam each rotate a certain angle, and then drives the cam slide out. So as to realize the control of the cam slide in the whole.

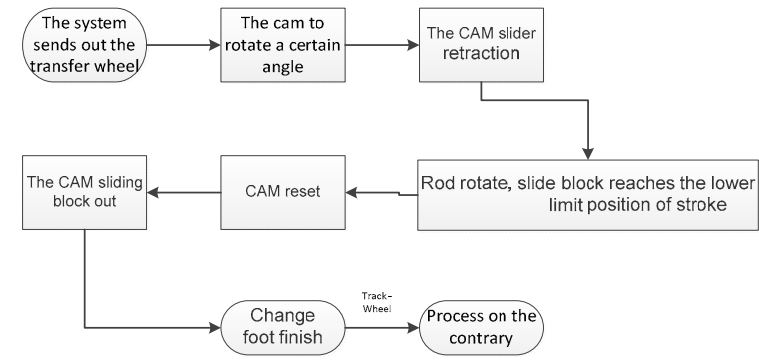

Figure 11 flow chart of overall change of leg

\section{Change of leg mechanism design part}

Through the above analysis, detection robot model should have the following advantages:

a) To realize the automatic detection of electromagnetic wave, and be able to work towards electromagnetic wave intensity maximum direction.

b) Mobile device with automatic.

c) The mobile device detection robot must adapt to various road conditions.

It has the following innovative points:

a) Using electromagnetic wave detecting robot within a certain range of the radiation intensity, and can automatically find the area of the intensity of the scope of the largest.

b) The product can move by itself, can avoid the direct contact of emission source, and solves the problem of measuring safety.

c) The robot can achieve the wheels and track free conversion, in order to adapt to the different geographical environment.

\section{Acknowledgment}

The work is supported by a teaching research project for Wuhan University of Technology (2013009) and a National Undergraduate Training Programs for Innovation and Entrepreneurship (20141049718005)

\section{References}

[1] Qi Hai Ming. Mobile robot tracer positioning technology research of Harbin Institute of Technology [D]. tube, 2010,06.

[2] Wang Bao. The design and implementation of [D].ARM\&Linux platform of Hunan University drainage pipeline inspection robot control system based on2011,03.

[3] Zhang Minfeng, Shen Zhongyu, dragon Chan. Biped robot DSP+ARM control system design of the [J]. in 2009 China intelligent automation conference proceedings based on,2009:832-836.

[4] Zhu Yansheng. All terrain Eight Legged Robot ARM control system programming based on 2013, 01.

[5] Zheng Xu. Design and implementation of [D]. Nanjing University of Science and Technology, pipeline inspection robot control system of 2013, 1 